\title{
Turnover of phosphatidic acid through distinct signaling pathways affects multiple aspects of pollen tube growth in tobacco
}

\section{Roman Pleskot ${ }^{1}$, Přemysl Pejchar ${ }^{1}$, Radek Bezvoda ${ }^{2}$, Irene K. LichtscheidI ${ }^{3}$, Mieke Wolters-Arts $^{4}$, Jan Marc $^{5}$, Viktor Žárský ${ }^{1,2}$ and Martin Potocký ${ }^{1}$ *}

1 Institute of Experimental Botany, v. v. i., Academy of Sciences of the Czech Republic, Prague, Czech Republic

2 Department of Experimental Plant Biology, Faculty of Science, Charles University in Prague, Prague, Czech Republic

${ }^{3}$ Core Facility of Cell Imaging and Ultrastructure Research, University of Vienna, Vienna, Austria

${ }^{4}$ Department of Molecular Plant Physiology, Institute for Wetland and Water Research, Radboud University Nijmegen, Nijmegen, Netherlands

${ }^{5}$ School of Biological Sciences, University of Sydney, Sydney, NSW, Australia

\section{Edited by:}

Xuemin Wang, University of Missouri, USA

\section{Reviewed by:}

Robert L. Houtz, University of Kentucky, USA

Serena Varotto, University of Padova, Italy

\section{*Correspondence}

Martin Potocký, Laboratory of Cell Biology, Institute of Experimental Botany AS CR, v.v.i., Academy of Sciences of the Czech Republic, Rozvojová 263, 16502 Prague 6, Lysolaje, Czech Republic. e-mail:potocky@ueb.cas.cz
Phosphatidic acid (PA) is an important intermediate in membrane lipid metabolism that acts as a key component of signaling networks, regulating the spatio-temporal dynamics of the endomembrane system and the cytoskeleton. Using tobacco pollen tubes as a model, we addressed the signaling effects of PA by probing the functions of three most relevant enzymes that regulate the production and degradation of PA, namely, phospholipases $D$ (PLD), diacylglycerol kinases (DGKs), and lipid phosphate phosphatases (LPPs). Phylogenetic analysis indicated a highly dynamic evolution of all three lipid-modifying enzymes in land plants, with many clade-specific duplications or losses and massive diversification of the C2-PLD family. In silico transcriptomic survey revealed increased levels of expression of all three PA-regulatory genes in pollen development (particularly the DGKs). Using specific inhibitors we were able to distinguish the contributions of PLDs, DGKs, and LPPs into PA-regulated processes. Thus, suppressing PA production by inhibiting either PLD or DGK activity compromised membrane trafficking except early endocytosis, disrupted tip-localized deposition of cell wall material, especially pectins, and inhibited pollen tube growth. Conversely, suppressing PA degradation by inhibiting LPP activity using any of three different inhibitors significantly stimulated pollen tube growth, and similar effect was achieved by suppressing the expression of tobacco pollen LPP4 using antisense knock-down. Interestingly, inhibiting specifically DGK changed vacuolar dynamics and the morphology of pollen tubes, whereas inhibiting specifically PLD disrupted the actin cytoskeleton. Overall, our results demonstrate the critical importance of all three types of enzymes involved in PA production and degradation, with strikingly different roles of PA produced by the PLD and DGK pathways, in pollen tube growth.

Keywords: phosphatidic acid, pollen tube, phospholipase D, diacylglycerol kinase, lipid phosphate phosphatase, tobacco, signaling, tip growth

\section{INTRODUCTION}

The pollen grain of flowering plants germinates on a receptive stigma and develops a pollen tube that grows through the pistil toward the ovules, thus serving as a courier that delivers the sperm cells to the embryo sac. For successful fertilization to occur, the pollen tube must accomplish two main goals - to elongate effectively and to navigate its way in the desirable direction. To achieve this, pollen tubes perform a specialized type of cell expansion called "tip growth" in which all growth occurs in a single region, the apical part of the tip-growing cell. Besides pollen tubes, other cell types such as root hairs, fungal hyphae, and neuronal cells likewise elongate by tip growth, sharing conserved mechanisms that bring about this type of growth. A crucial aspect of tip growth is the establishment of a unique growth domain at the plasma membrane, the maintenance of which and also the accompanying membrane trafficking require appropriately regulated lipid signaling (reviewed in Malhó et al., 2006; Žárský and Potocký, 2010; Zonia, 2010).

Lipid signaling employs various messenger molecules that carry out communication between the plasma membrane, the endomembrane compartments, and the cytoplasm. This communication system is based on specific lipids as messengers and enzymes responsible for their production and degradation. Phosphoinositides (PPIs) together with various kinases and phosphatases were described as specific lipids and enzymes with signaling functions earlier (Martin, 1998). In addition to PPIs, the simplest phospholipid, phosphatidic acid (PA), has emerged as an important signaling molecule that plays a fundamental role in a variety of biotic and abiotic stresses such as wounding, pathogen attack, salinity, drought, and cold (Bargmann and Munnik, 2006; 
Testerink and Munnik, 2011). Moreover, PA has been shown to play a critical role in the regulation of tip growth in pollen tubes and root hairs (Ohashi et al., 2003; Potocký et al., 2003; Monteiro et al., 2005). Two major, distinct signaling pathways contribute to the production of PA (Kooijman and Testerink, 2010). One is a direct cleavage of structural phospholipids by phospholipase $\mathrm{D}$ (PLD), and another is a sequence of reactions catalyzed by phospholipase C (PLC) and diacylglycerol kinase (DGK). Cellular PA levels may be attenuated either through dephosphorylation to diacylglycerol (DAG) by PA phosphatase (reviewed in Nakamura and Ohta, 2010) or through phosphorylation to diacylglycerol pyrophosphate (DGPP) by a PA kinase that awaits molecular characterization (van Schooten et al., 2006). PA acts as a membrane-localized signal, affecting downstream responses by binding specific protein targets including a variety of protein kinases, phosphatases and specific proteins involved in vesicle trafficking or regulation of the actin cytoskeleton (Testerink and Munnik, 2011). As a structural membrane lipid, PA also affects the biophysical properties of the plasma membrane because of its negative charge and a cone- like shape, which promotes membrane curvature (Kooijman et al., 2003; Testerink and Munnik, 2011).

Phospholipases D are ubiquitous eukaryotic enzymes that cleave structural phospholipids, releasing PA and a free polar headgroup. Plants employ two distinct subfamilies of canonical PLDs, a C2-domain-containing subfamily and the PX- and PH-domaincontaining subfamily. Moreover, the C2-domain-containing subfamily forms at least five different subclasses with specific biochemical and functional properties. We have shown that PLD activity is crucial for the regulation of pollen tube growth (Potocký et al., 2003; Pleskot et al., 2010).

In the alternative pathway of PA production, PLC first produces DAG by cleaving either phosphatidylinositol $(4,5)$-bisphosphate (PI-specific PLCs) or structural phospholipids (non-specific PLCs). DAG can be subsequently phosphorylated by DGK to PA. The main signature of DGKs is the presence of two domains, the DGK catalytic motif and the DGK accessory kinase motif. Moreover, some DGK isoforms, mainly those in metazoa, contain additional regulatory motifs, including $\mathrm{C} 1$ domain, calciumbinding EF hand motif, ankyrin repeats, $\mathrm{PH}$-domain, and others. Although DGK was originally described in the 1980s and the first human DGK was cloned in the 1990s (for review see Mérida et al., 2008) only a handful of more recent studies have described the role of DGKs in plants, mainly in the context of various stress responses (Ruelland et al., 2002; GómezMerino et al., 2004, 2005; Arisz et al., 2009; Arisz and Munnik, 2011).

As for any signaling mediator, PA levels must be appropriately regulated to achieve temporal and spatial control of signaling. For down-regulation, the activity of PA phosphatase can be mediated by genes from two different families, lipins and lipid phosphate phosphatases (LPPs). Both families have homologs in mammals, fungi, and plants. Lipin activity is $\mathrm{Mg}^{2+}$-dependent and PA-specific (Donkor et al., 2007). The Arabidopsis genome contains two lipin homologs, Pah1 and Pah2, which seem to act in the eukaryotic pathway of glycerolipid metabolism, especially during phosphate depletion. The two proteins provide
DAG for further utilization in the inner membranes of plastids (Nakamura et al., 2009). LPPs are membrane-bound proteins that contain six transmembrane domains and three conserved motifs. LPPs have broader substrate specificity than lipins, with some isoforms utilizing both DGPP and PA, whereas others have a strong preference for a particular phospholipid. Arabidopsis has four LPP genes with clear homology to mammalian and yeast genes. AtLPP1 is a DGPP-preferring enzyme induced by stresses and elicitors, whereas AtLPP2 shows no preference for either DGPP or PA. AtLPP3 and AtLPP4 remain to be characterized (Nakamura and Ohta, 2010). Arabidopsis genome also encodes five genes that share several conserved amino-acid residues with canonical eukaryotic LPPs and are homologous to cyanobacterial genes (therefore termed prokaryotic LPPs). Three of these are localized to plastids where they are thought to play a role in general lipid metabolism (Nakamura et al., 2007).

To date, only the activity of PLD has been studied in the context of pollen tube tip growth (Potocký et al., 2003; Monteiro et al., 2005) while there are no data on the role of DGK or LPP. Since these enzymes belong to multi-gene families, it is advantageous to use pharmacology to investigate the general role of the entire enzyme family. In the present work, we employed this approach to study the turnover of PA mediated by the PLD, DGK, and LPP pathways in tobacco pollen tubes. We used inhibitors that are known to affect specifically different enzymes involved in PA signaling. In particular, we used $n$-butanol ( $n$-ButOH) to inhibit PLD-produced PA (Ella et al., 1997; McDermott et al., 2004), the compound R59022 to inhibit DGK (Ruelland et al., 2002; Gómez-Merino et al., 2004, 2005) and several known inhibitors of eukaryotic LPP activity such as NEM, PG, and propranolol (Meier et al., 1998; Furneisen and Carman, 2000). We show that these inhibitors have distinct effects on the growth of the pollen tube as well as on the morphology and the dynamics of various cellular structures. The results indicate that distinct PA signaling pools are present in the tip of a growing pollen tube where they modulate various cellular processes.

\section{RESULTS}

\section{THE EVOLUTION OF KEY PA SIGNALING GENES SHOWS A HIGH DIVERSITY IN THE PLANT KINGDOM}

In order to elucidate the pattern of PA signaling in pollen tubes, we analyzed the evolution and gene expression of PLDs, DGKs, and LPPs, focusing on multicellular "higher" plants with cells that exhibit tip growth.

Plant PLDs comprise the most expanded group from all the genes analyzed. This family can be divided into two well-supported clades corresponding to the C2-PLD and PXPH-PLD subfamilies (Figure 1A). Our initial sequence analysis suggested the $\mathrm{C} 2$ PLDs form two main classes, named after the first-characterized members, namely, the PLD $\alpha$-like class and the $\beta / \gamma / \delta$-class (Eliáš et al., 2002). These two classes differ also in their gene structure, with the $\alpha$-class genes usually consisting of three or four exons whereas genes of the $\beta / \gamma / \delta$-class have 10 exons. Our data show that this diversification is probably conserved across all land plants since members of both classes are found in Physcomitrella and Selaginella. In the $\beta / \gamma / \delta$-class there are four moss genes and 
two lycophyte genes, and the $\alpha$-class contains three genes from Selaginella and five genes from Physcomitrella. Exon-intron structure and all conserved sequence blocks are well preserved for both classes among angiosperm, moss, and lycophyte genes. Unexpectedly, Physcomitrella and Selaginella PLDs do not form the most basal branches within the 3-exon or 10-exon C2-PLDs but instead cluster together with conventional PLD $\alpha$ and PLD $\delta$ subclasses, respectively (with the exception of PpatPLD $\alpha 5$ ).

Both Selaginella and Physcomitrella PLDs further multiplicated independently after the separation of mosses, lycophytes, and seed plants. Rapid diversification of C2-PLDs is evident also from the evolution of angiosperm orthologs. In eudicots, some subclasses originally defined in Arabidopsis are well conserved in all analyzed species. Thus, homologs of PLD 1-2, $P L D \alpha 3, P L D \epsilon, P L D \beta 1-2$, and $P L D \delta$ are found also in poplar and Medicago, although multiple duplications within these clades are frequently observed. Conversely, other PLD subclasses have an uneven distribution, for example, 10-exon PLD class named $P L D v$ and defined originally for rice has clear orthologs in both poplar and Medicago but not in Arabidopsis. On the other hand, no members of the PLD $\gamma$-subclass could be found outside the Brassicaceae. As mentioned above, one moss PLD gene (PpatPLD $\alpha 5$ ) has been placed outside the typical PLD $\alpha$-class but significant bootstrap support is lacking and neighbor-joining analysis suggested different topology (data not shown).

In contrast to the C2-type PLDs, plant PXPH-type PLDs represent a much smaller subfamily showing a distribution that corresponds to species evolution, with moss and lycophyte sequences occupying the base of the subtree followed by the angiosperm branch (Figure 1A). Most of the examined species contain two PXPH-type PLD genes. The ancestor land plant probably had one copy of the PXPH-PLD in the genome and duplications occurred independently after the diversification in the lines leading to mosses, lycophytes, eudicots, and monocots.

Similarly to the plant PLDs, plant DGKs also form two distinct subfamilies. Members of the first subfamily contain a predicted transmembrane helix and two $\mathrm{C} 1$ domains in addition to the catalytic motifs. Genes in this C1-type DGK class cluster into two well-supported classes. Selaginella DGK1 is located inside the tree, similarly to the evolution of the C2-PLDs. Surprisingly, we have not found a C1-type DGK in the genome of Physcomitrella patens. The second subfamily of DGKs contains just the catalytic motifs and is more expanded than the C1-type DGK. This subfamily also forms two classes but generally with much more clear relationships following organismal evolution, with Physcomitrella and Selaginella DGKs forming the basal branches of the evolutionary tree (Figure 1B).

Plant canonical LPPs form a small-size family of very highly conserved genes (Figure 1C). Generally, LPP phylogeny follows plant evolution, with many independent duplications and losses in different species. We found five LPP-encoding genes in the genome of Physcomitrella, which forms the base of the phylogenetic tree of plant LPPs together with three genes from Selaginella. However, the relationship between these basal clades is not clear because of a lack of statistical support. Four Arabidopsis LPP genes cluster into two separate clades together with other investigated eudicots

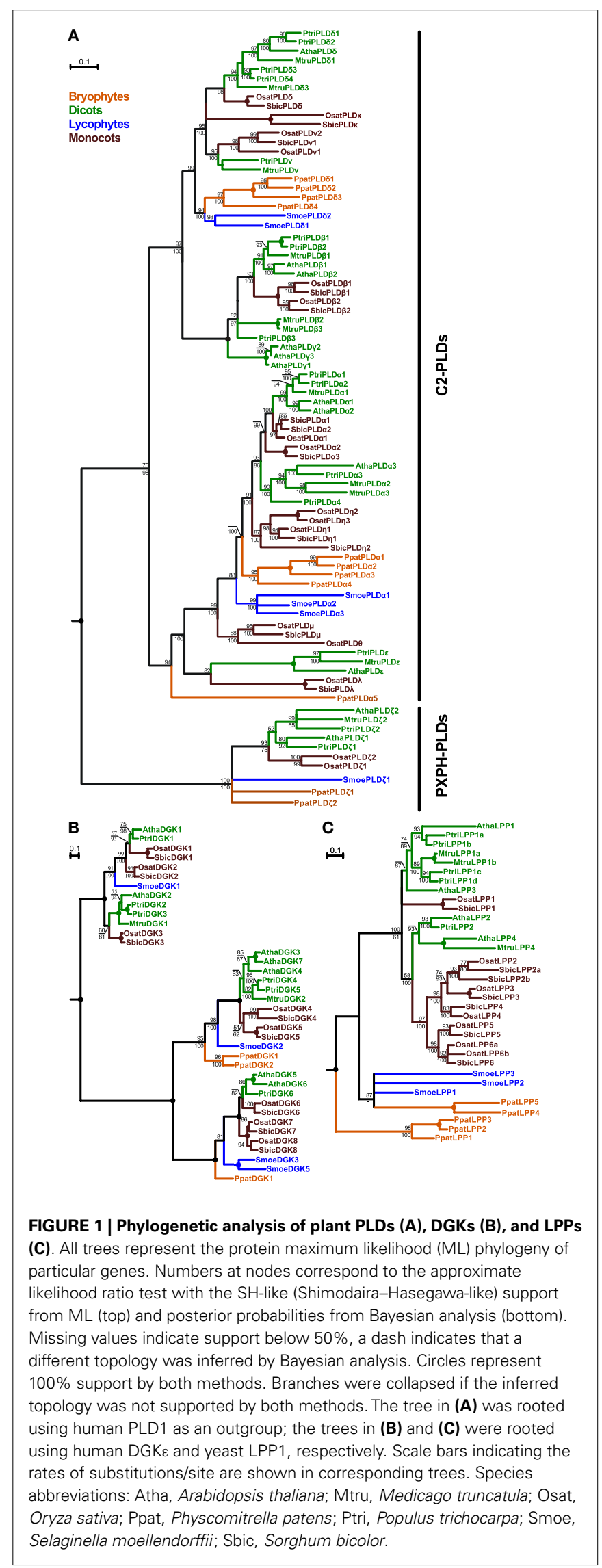


LPP genes including Populus and Medicago. Monocot genes also form two clades, one with a high statistical support and another one with unresolved relationship to other sequences.

\section{EXPRESSION ANALYSIS OF ARABIDOPSIS GENES ENCODING PLDS, DGKs, AND LPPs}

Given that our work focuses on the role of PA in the regulation of pollen tube growth, we were interested in the expression levels of genes coding for PLD, DGK, and LPP in various plant organs as well as pollen. We used the Genevestigator tool ${ }^{1}$ to obtain these data for Arabidopsis (Figure 2A). Intriguingly, most of the genes coding for DGK and LPP are strongly expressed in pollen, and two genes, DGK4 and LPP4, appear to be pollenspecific. Although only a half of the genes from the PLD family are expressed in pollen, four genes are strongly expressed. PLD $\alpha 2$, $\beta 1$, and $\delta$ are almost exclusively present in pollen and $P L D \xi 1$ is widely expressed throughout all studied organs. Similar observations can be drawn from the analysis of rice pollen transcriptome (Wei et al., 2010). Furthermore, the available pollen proteomic data show very good agreement between PLD, DGK, and LPP genes transcribed in pollen and the presence of translated proteins (Figure 2B). Together, these data indicate possible functional conservation of these genes in flowering plants. This is also corroborated by our previous finding showing a similar pattern of expression of C2-PLDs in tobacco (Pleskot et al., 2010).

\section{PHARMACOLOGICAL MANIPULATION OF PA LEVELS SHOWS THE INVOLVEMENT OF PLD, DGK, AND LPP PATHWAYS IN POLLEN TUBE TIP GROWTH}

The discovery of PA as a signaling molecule that controls polar expansion of pollen tubes (Potocký et al., 2003) rises the question of the potential target(s) of PA action. We employed available inhibitors of the key enzymes in PA production/degradation pathways to study potential distinct PA signaling pools and their likely targets. We used $n$-ButOH as an inhibitor of PLD-produced PA, R59022 as an inhibitor of DGK, and NEM, PG, and propranolol as inhibitors of PA phosphatase activity. Initially, we were interested if these inhibitors have an effect on pollen tube growth. Identically to our previous results (Potocký et al., 2003), we observed that $n$ ButOH inhibited pollen tube growth in concentration-dependent manner. Similarly, we found a strong, concentration-dependent inhibition of tobacco pollen tube growth after application of the DGK inhibitor R59022. Even the lowest concentration of R59022 $(25 \mu \mathrm{M})$ caused a dramatic inhibition of pollen tube growth to approximately $30 \%$ of that in controls (Figure 3A). In striking contrast, applications of the various LPP inhibitors promoted the growth of the pollen tubes (Figures 3B-D), similarly to the promontory effect of exogenously added PA that we showed previously (Potocký et al., 2003). Furthermore, a short pre-treatment of pollen tubes with either PG or NEM counteracted growth arrest mediated by $n$-ButOH (data not shown).

In subsequent experiments, we used $0.5 \% n$-ButOH, $50 \mu \mathrm{M}$ R59022, $50 \mu \mathrm{M}$ dioctanoyl-PA, and $50 \mathrm{nM}$ NEM. For all experiments, additional control treatments with $0.5 \% t$-ButOH or

\footnotetext{
${ }^{1}$ www.genevestigator.ethz.ch
}

DMSO were performed. Since they were indistinguishable from cells treated with medium alone, for the sake of brevity, we omitted them from the figures.

\section{REDUCTION IN PA LEVEL DISRUPTS MEMBRANE TRAFFICKING BUT NOT EARLY ENDOCYTOSIS}

Given that for tip-growing cells vesicular traffic is crucial for the delivery of cell wall-building material to the apex, we tested the effect of inhibitors of PA signaling on this process. We employed the amphiphilic styryl dye FM 1-43, which has been used successfully to monitor endo- and exocytosis in Lilium and Agapanthus pollen tubes (Parton et al., 2001; Camacho and Malhó, 2003). The FM 1-43 staining pattern in control tobacco pollen tubes was strikingly similar to the reported images, indicating that the FM 1-43 dye is suitable for monitoring endomembrane trafficking in our experiments (Figure 4).

To test the involvement of PA turnover in early endocytosis, we examined the effect of $n$-ButOH, R59022, and NEM on internalization of FM 1-43. After 10 min incubation with $2 \mu \mathrm{M}$ FM 1-43 on ice, fluorescent signal was located uniformly along the pollen tube plasma membrane (data not shown). Regardless of treatment, we have not observed any difference in internalization of FM 1-43 during the first $1.5 \mathrm{~min}$, when almost immediately mobile intracellular spots, presumably representing components of early endosomal machinery, started to appear in the subapical region (Figure 4A). In control cells, the dye was initially localized to

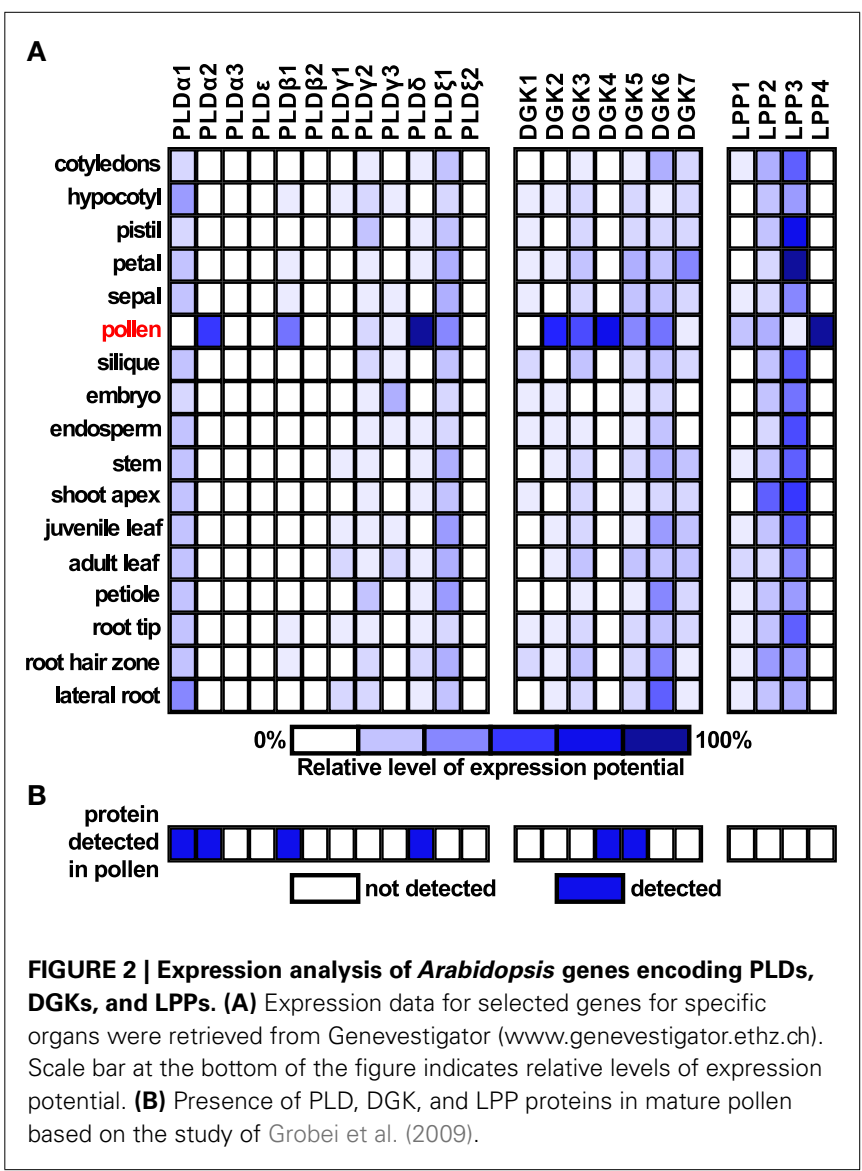



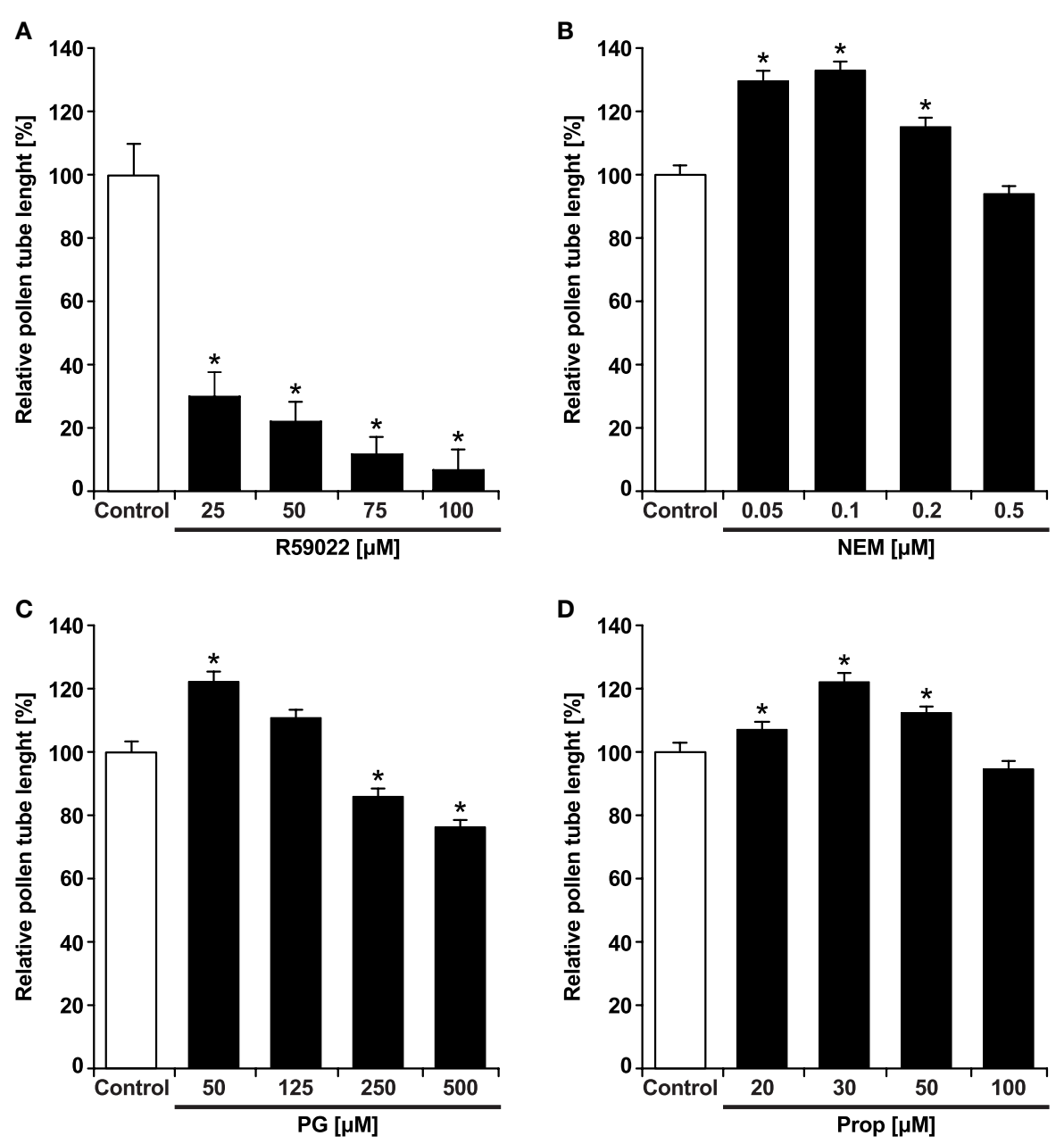

FIGURE 3 | The DGK inhibitor R59022 inhibits pollen tube growth, whereas the LPP inhibitors NEM, PG, and propranolol promote pollen tube growth. Pollen tubes were incubated for $90 \mathrm{~min}$ in germination medium supplemented with specified concentrations of R59022 (A), NEM (B), PG (C), and propranolol (D). The pollen tubes were then fixed with $3.7 \%$

endocytic compartments, although an inverted-cone-like staining pattern, presumably consisting of endo- and exocytic vesicles, was formed at the cell tip within $20 \mathrm{~min}$. A similar pattern was observed in cells treated with NEM or PA. In contrast, pollen tubes treated with either $n$-ButOH or R59022 never formed an inverted-cone, but instead the FM 1-43 dye accumulated along the sides of the tube in the subapical region about $10-15 \mu \mathrm{m}$ back from the cell tip. This pattern became even more pronounced over time. Interestingly, this pattern corresponds to the zone of maximal clathrin-coated endocytosis as described by Derksen et al. (1995) and Moscatelli et al. (2007).

We also tested the involvement of PA signaling in intracellular membrane trafficking after an extended FM 1-43 staining, which also labels exocytotic events (Zonia and Munnik, 2008). The cells were first incubated with FM 1-43 for 90 min and then treated with a drug for $10 \mathrm{~min}$, after which the distribution of the signal was examined (Figure 4B). In this case, we again saw no difference between control cells and application of either NEM or PA. formaldehyde and the length of pollen tubes was measured. At least 120 cells were measured for each treatment in two independent experiments. Data represent the average pollen tube length \pm SEM. Significant difference from the control samples is indicated by an asterisk (ANOVA, Kruskal-Wallis test, $p<0.05)$.
However, both $n$-ButOH and R59022 again caused the loss of the inverted-cone and accumulation of the dye along the sides of the tube in the subapical region.

\section{SUPPRESSION OF PA SIGNALING ALTERS THE DEPOSITION OF PECTINS AND CALLOSE AT THE CELL WALL}

Since the modulation of PA levels resulted in profound changes in membrane trafficking, and given the finding that the cell wall at the growing tip is mainly formed by secreted pectins (Geitmann and Steer, 2006), we employed the Ruthenium red dye (Ischebeck et al., 2008) to study the effect of inhibitors of PA signaling on the deposition of pectins. In control cells, Ruthenium red labeled strongly the very tip (Figure 5A). In cells treated with NEM and PA, there was an increase in the intensity of the apical signal (Figures 5A,B), suggesting an enhanced secretion of pectins after PA elevation. In contrast, $n$-ButOH and R59022 caused a complete loss of the apical signal. Moreover, in the case of R59022, we consistently found large punctuate structures 


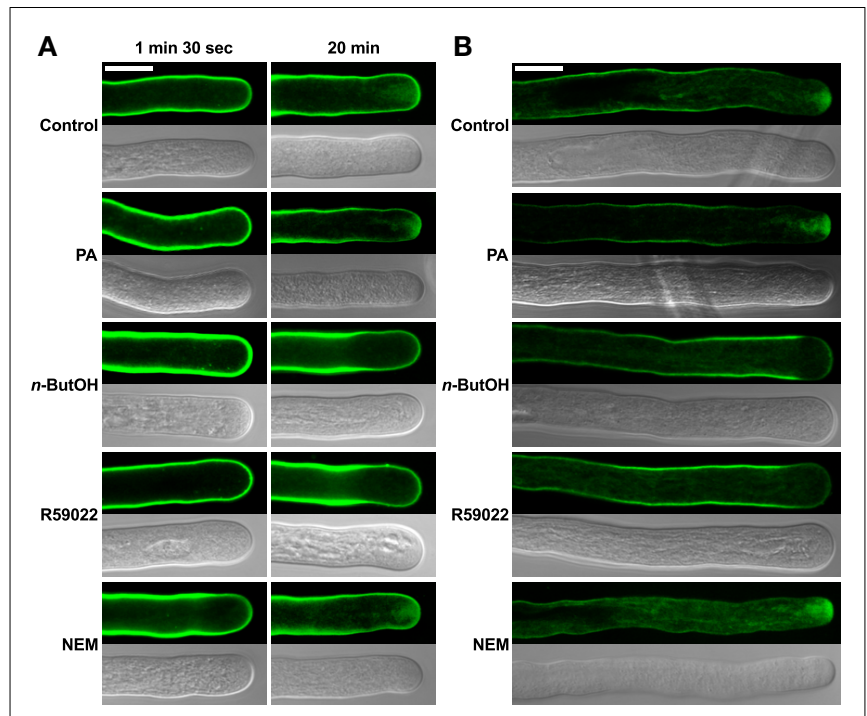

FIGURE 4 | The PLD antagonist $\boldsymbol{n}$-ButOH and DGK inhibitor R59022 disrupt internalization of the endomembrane tracer dye FM 1-43. (A) Ninety-minute-old pollen tubes were pre-incubated in germination medium on ice for $10 \mathrm{~min}$, and $2 \mu \mathrm{M}$ FM1-43 dye together with a particular drug or PA were then simultaneously added and the cells monitored for $30 \mathrm{~min}$. Representative pollen tubes observed at two times are shown. (B) Alternatively, pollen tubes were incubated in germination medium containing $2 \mu \mathrm{M}$ FM1-43 for 90 min, a particular drug or PA were then added for $10 \mathrm{~min}$ and the cells were observed with a confocal microscope. At least 15 cells were analyzed for each treatment in four independent experiments and typical examples are shown. Scale bar $=10 \mu \mathrm{m}$.

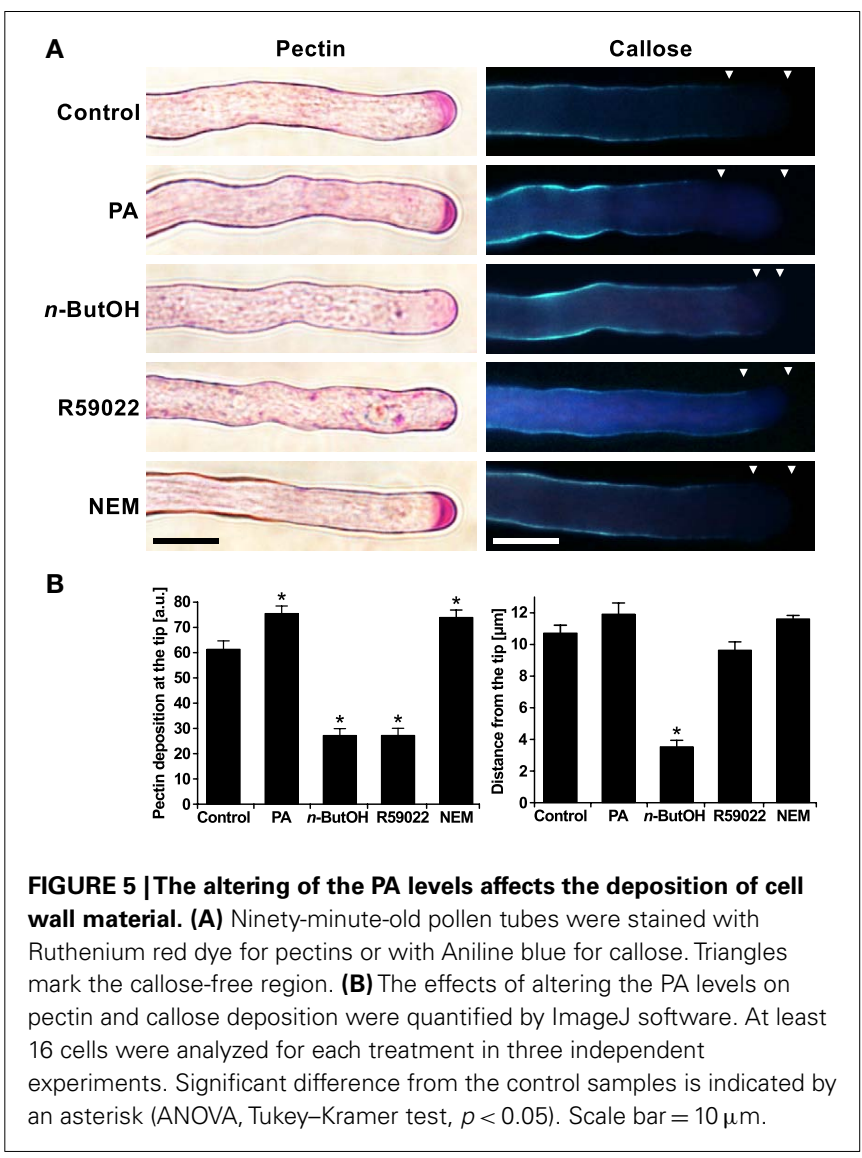

vacuolar thread-like strands but these strands protruded to the apical region, which we have not seen in the controls. Intriguingly, R59022 caused a dramatic change in the vacuole morphology, with the vacuolar strands aggregated and protruding toward the apex. The vacuolar aggregates were also visible in the light microscope using Nomarski optics.

\section{ACTIN POLYMERIZATION IS SPECIFICALLY REGULATED BY PLD-DERIVED PA}

We previously identified a relationship between actin microfilaments and PLD-formed PA, and described a direct interaction between actin and tobacco PLD $\beta 1$, which is specific for this process (Pleskot et al., 2010). To examine a putative involvement of the PLC/DGK pathway in the regulation of actin dynamics, here we treated pollen tubes with PLD, DGK, and LPP inhibitors and then visualized the actin cytoskeleton by Alexa Fluor 633 Phalloidin labeling according to Pleskot et al. (2010). Control cells exhibited long actin cables oriented longitudinally in the shank and a fringe-like structure in the subapical region (Figure 7), similar to published observations (Lovy-Wheeler et al., 2005; Pleskot et al., 2010). Treatment with $0.5 \% n$-ButOH for $20 \mathrm{~min}$, however, led to a dramatic disintegration of actin filaments (Figure 7; Pleskot et al., 2010); the effect of $n$-ButOH was very fast, causing distinct actin disruption already within $5 \mathrm{~min}$ of application. Treatment with R59022 for 20 min did not affect actin organization. Application of NEM often increased the intensity of the actin signal, similarly to the effect of applied PA (Pleskot et al., 2010). 


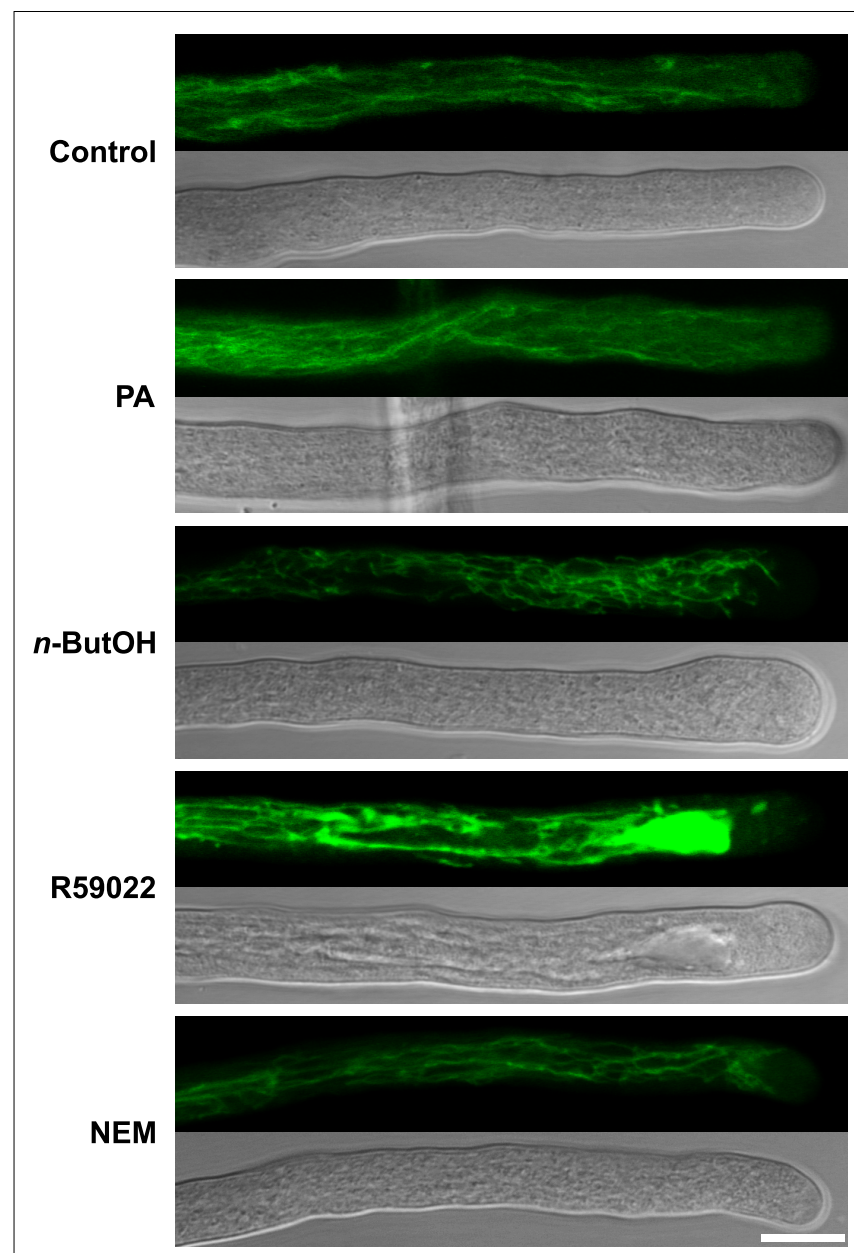

FIGURE 6 | Pharmacological inhibition of DGK causes changes in vacuolar morphology. Ninety-minute-old pollen tubes were stained with the vacuolar marker $1 \mu \mathrm{M}$ carboxy-DCFDA for $5 \mathrm{~min}$ and then transferred either to a control medium or media containing a particular drug or PA for $15 \mathrm{~min}$. Subsequently, the pollen tubes were imaged with a confocal microscope. At least 20 cells were analyzed for each treatment in four independent experiments and typical examples are shown. Scale bar $=10 \mu \mathrm{m}$

\section{PLD INHIBITION AFFECTS POLLEN TUBE CYTO-ARCHITECTURE AND INTRACELLULAR DYNAMICS}

It has been suggested that the spatio-temporal flow of secretory vesicles in tip-growing pollen tubes is regulated by tightly tuned actin dynamics and organization (Kroeger et al., 2009). Since we have demonstrated the role of PLD-derived PA in the regulation of actin polymerization, our next step was to investigate the connection between PLD-derived PA and particle movement in the growing tip. To study this in real time, we used video-enhanced contrast microscopy (Ovečka et al., 2008). In control tobacco pollen tubes, we observed the typical cyto-architecture with a V-shaped tip region $\sim 4 \mu \mathrm{m}$ long and free of larger organelles (Figure 8A ). Most of this clear-zone was filled with rapidly moving particles which were hardly discernible on still images. The motion of larger organelles was slower and displayed typical reverse fountain-like movement.

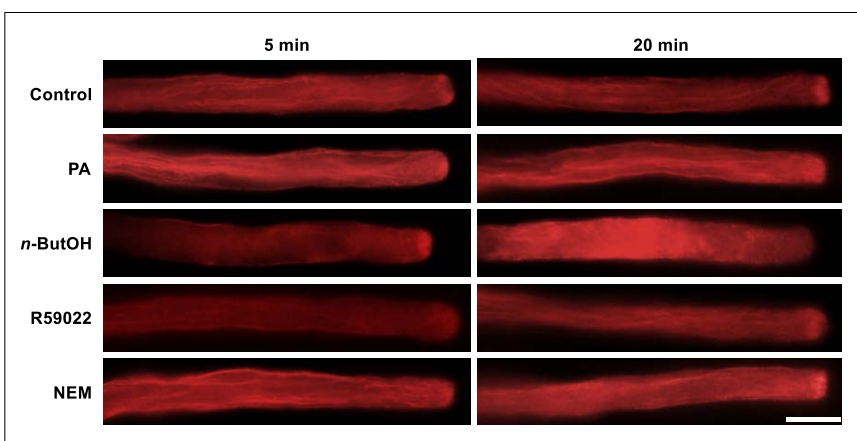

FIGURE 7 | PLD-derived PA is specifically involved in the regulation of the actin dynamics. Pollen tube cultures $(90 \mathrm{~min}$ after imbibition in sucrose medium) were treated with a particular drug, PA or the corresponding volume of control solution for 5 or $20 \mathrm{~min}$ before fixation. The actin cytoskeleton was visualized by Alexa Fluor 633 Phalloidin as described in Pleskot et al. (2010). At least 20 cells were analyzed for each treatment in three independent experiments and typical examples are shown. Scale bar $=10 \mu \mathrm{m}$

Intriguingly, when the cells were supplied with medium alone, within $4 \mathrm{~min}$ after addition we routinely observed rapid, partial loss of the pollen tube polarity. This depolarization was transient and lasted for only $\sim 4-6 \mathrm{~min}$. Typically, the pollen tube underwent partial swelling, followed by a period of slower growth and then normal tip polarity and growth rate resumed, occasionally establishing a new growth direction. Similar behavior was sometimes observed also in cells without any treatment, indicating that mechanical stress caused by transfer of pollen tubes from culture to microscope slide was the likely source of the transient depolarization.

In striking contrast, addition of the PLD inhibitor $n$-ButOH did not invoke cell depolarization but instead resulted in rapid retardation and eventually a complete stop of pollen tube growth (Figures 8A,B). The clear-zone gradually diminished and the larger organelles invaded the tip region. It is noteworthy that cytoplasmic streaming continued in $n$-ButOH-treated pollen tubes in a manner indistinguishable from streaming in controls. A similar pattern of growth cessation was seen after the addition of R59022 (Figure 8B and data not shown).

The addition of $50 \mu \mathrm{M}$ PA swiftly boosted the elongation of tobacco pollen tubes, suggesting an almost instant effect of PA on elongation rates (Figures 8A,B). We were unable to image pollen tubes earlier than $2 \mathrm{~min}$ after the treatment because of technical difficulties when attempting experiments with direct perfusion of PA into pollen tubes. Interestingly, unlike control cells, pollen tubes treated with PA did not undergo the transient depolarization seen in the controls and maintained an undisturbed morphology, suggesting that elevated concentration of PA somehow protected the cell from stress-induced depolarization.

Having established the existence of rapid changes in the clearzone after $n$-ButOH or PA treatments, we then attempted to analyze the intracellular movement of particles in the apical zone. In control cells, the particles moved with average velocities of $\sim 2.5 \mu \mathrm{m} / \mathrm{s}$ (Figure 9A). This value is close to the results obtained for Picea pollen tubes (Wang et al., 2006) and tobacco root hairs 
(Šamaj et al., 2002). The movement rates in control cells remained constant during the experiment. We were unable to distinguish any prevalent direction of particle movement, which would require the collection of a large amount of data to form a clear conclusion. In contrast to control cells, addition of either $0.5 \% n$-ButOH or $50 \mu \mathrm{M}$ PA caused significant changes in the dynamics of particles at the tip. PLD inhibition caused progressive slowing-down of movement of the particles, which often resembled random oscillations. On the other hand, addition of PA resulted in fast acceleration of the tip-localized particles within $2 \mathrm{~min}$, reaching an average velocity of $3.2 \mu \mathrm{m} / \mathrm{s}$. This effect was restricted to the apical region; when we measured velocities of the large organelles in the shank we did not see any effect (Figure 9B). Collectively, these data suggest that manipulating PLD-derived PA levels affects the growth rate of the pollen tube by regulating the dynamics of the particles in the apex.

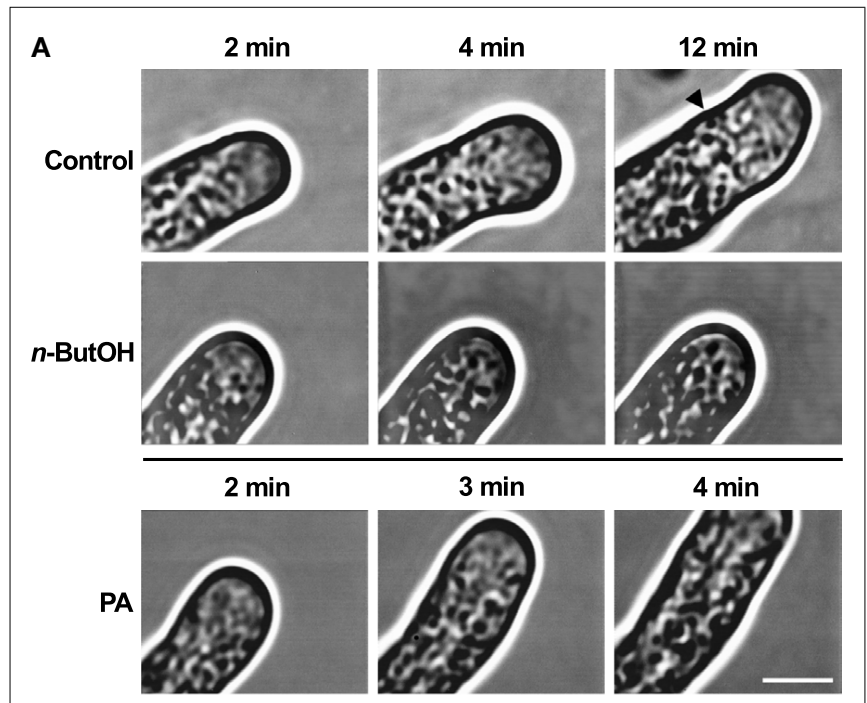

B

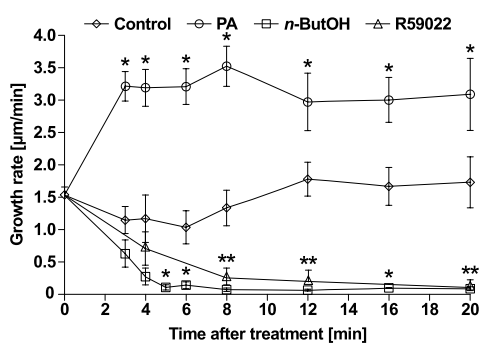

FIGURE 8 | Alterations in PA levels in tobacco pollen tubes result in changes in the growth and architecture of the cell. (A) Time-series analysis of pollen tubes treated with $n$-ButOH or PA by video-enhanced contrast microscopy. Ninety-minute-old cells were treated with $0.5 \%$ $n$-ButOH, $50 \mu \mathrm{M} \mathrm{PA}$, or control medium and imaged after indicated times. The image field for control cells had to be shifted after $8 \mathrm{~min}$ of imaging and the starting position of the pollen tube tip after the shift is marked. At least six pollen tubes were imaged for each treatment and representative pictures are shown. (B) Growth rates of cells after treatments with $n$-ButOH, R59022 or PA. Data points represent the mean values of at least six pollen tubes \pm SEM. Significant difference from the control samples is indicated by an asterisk (ANOVA, Tukey-Kramer test, $p<0.05$ ). Scale bar $=5 \mu \mathrm{m}$
Complementary to the analysis of living cells, we examined the effect of PLD inhibition on pollen tubes at ultrastructural level. Although the ideal method for fixation of highly dynamic cells would be freeze fixation/substitution, unfortunately pollen tubes incubated with primary or tertiary alcohols necessary for the procedure showed burst tips. We therefore used the traditional, albeit more artifact-prone chemical fixation. Nevertheless, Figure 10 shows that control samples display all characteristic features of normally growing pollen tubes. These include the typical shape of the apical dome, accumulation of secretory vesicles at the apex that form the typical "inverted-cone" structure, and exclusion of larger organelles like mitochondria and Golgi bodies from the very tip.

The ultrastructural features of cells incubated for $15 \mathrm{~min}$ in $0.5 \% n-\mathrm{ButOH}$ are consistent with the findings from the videoenhanced contrast microscopy. Most notably, the number of secretory vesicles in the tip is dramatically reduced (Figure 10B), typical zonation of organelles in the pollen tube is lost, and mitochondria together with other big organelles are relocated to the extreme apex. Moreover, a thickened cell wall could be observed in the tip region of $n$-ButOH-inhibited cells. Recently, the ultrastructure of Agapanthus pollen after PLD inhibition was reported (Monteiro et al., 2005). The authors also observed loss of secretory vesicles from the tip and bigger organelles protruding toward the apex. In contrast, massive swelling of Agapanthus pollen tubes induced by $n$-ButOH was not observed in tobacco. This discrepancy may result from experimental differences since Monteiro et al. (2005) used $30 \mathrm{~min}$ incubation in $0.75 \% n$-ButOH, or it may reflect species differences.

\section{KNOCK-DOWN OF TOBACCO POLLEN LPP GENE EXPRESSION PROMOTES POLLEN TUBE GROWTH}

To further confirm the outcomes of the pharmacological approach, we employed antisense knock-down strategy which has been successfully used by our group and others to specifically knock-down
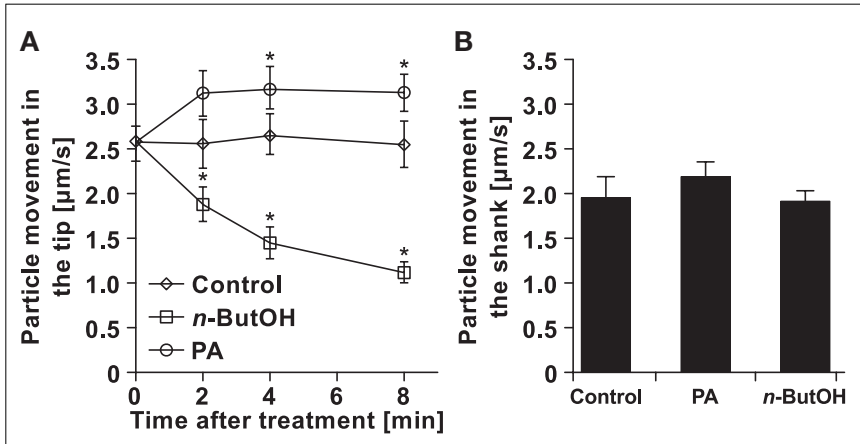

FIGURE 9 | Changes in particle dynamics in pollen tubes in response to altered PA levels. (A) Analysis of particle movement in the tips of pollen tubes treated with $n-\mathrm{ButOH}, \mathrm{PA}$, or control medium. Three pollen tubes were selected from each treatment and the movement of individual particles in the tip was followed for total time of $500 \mathrm{~ms}$ in $~ 80 \mathrm{~ms}$ intervals using the Manual Tracking plugin of the ImageJ software. (B) Analysis of movements of big organelles in the shank of the same pollen tubes as in (A) was done after $8 \mathrm{~min}$. Data represent the mean values of 14-19 trajectories \pm SEM. Significant difference from the control samples is indicated by an asterisk (ANOVA, Tukey-Kramer test, $p<0.05$ ). 


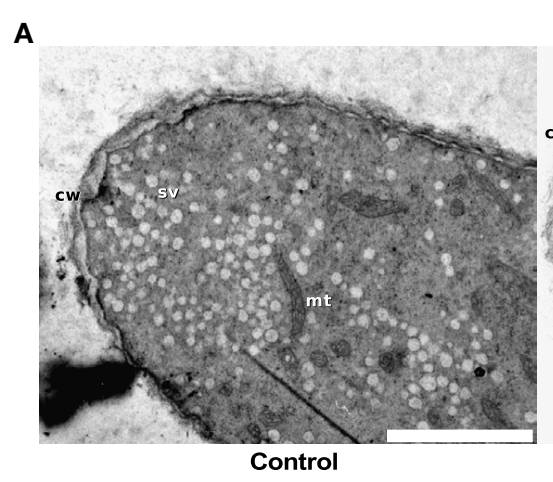

FIGURE 10 | PLD inhibition causes changes in the ultrastructure of the pollen tube tip and decreases the number of secretory vesicles. The micrographs in (A) show the ultrastructure of a control pollen tube and a cell treated with $0.5 \% n$-ButOH for 15 min before chemical fixation. $\mathrm{cw}$, cell wall; $\mathrm{mt}$, mitochondrion; sv, secretory
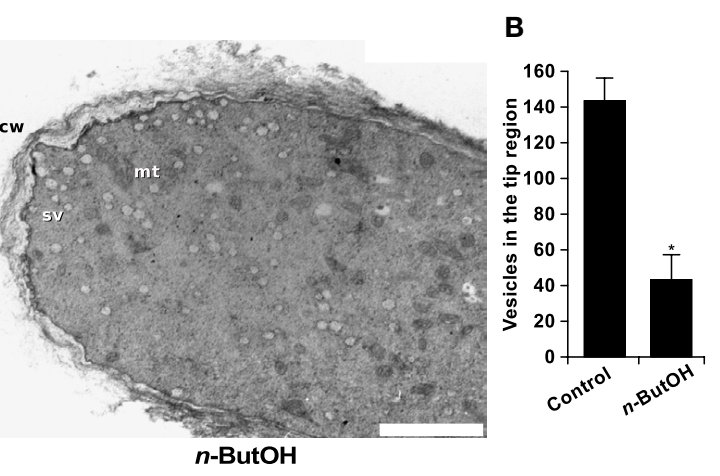

vesicle. Scale bar $=2 \mu \mathrm{m}$. (B) Quantification of secretory vesicles in the tip region. Three cells were analyzed for each treatment; data represent mean values \pm SEM. Significant difference from the control sample is indicated by an asterisk (ANOVA, Tukey-Kramer test, $p<0.05)$. gene expression in pollen tubes (Moutinho et al., 2001; Sun et al., 2005; Potocký et al., 2007; Pleskot et al., 2010). We took advantage of the fact that LPP expression in Arabidopsis pollen is restricted to the single isoform LPP4 (Figure 2) and cloned the homologous gene from tobacco pollen. We designed specific antisense oligodeoxynucleotides (ODNs) against this gene. Addition of liposomes containing these antisense ODNs into the imbibition medium promoted pollen tube growth (Figure 11), an effect similar to that obtained with the drug-induced inhibition of PA phosphatase activity described above (Figures 3B-D).

\section{DISCUSSION}

In this work, we elucidated the plant-focused evolution of several key enzymes in PA signaling, namely PLD, DGK, and LPP. Our phylogenetic analysis revealed massive diversification of the C2PLD family in land plants compared with DGK or LPP. Several, not mutually exclusive, hypotheses may explain this unexpected diversity in PLDs. First, independent multiplications may indicate that numerous PLD paralogs can evolve quickly to be engaged in many different signaling pathways required by the organism. The sessile nature of land plants may generate such demand, and various plant PLDs have indeed been implicated in responses to a number of biotic and abiotic stresses. Furthermore, the more conserved character of the PLC/DGK pathway compared with the PLD pathway that can be seen in many eukaryotes (data not shown) may indicate higher evolutionary flexibility of PLDs. Second, part of the PLD diversity in plants may be related to the demands of the cell polarity machinery that defines geometrically distinct cellular surfaces in the context of a multicellular body that consists of cells endowed with rather rigid cell walls. Several other genes involved in the regulation of cell polarity, such as the subunits of the membrane-tethering exocyst complex (Eliáš et al., 2003) or the actin nucleators formins (Grunt et al., 2008), are found in only one or a few copies in mammalian and yeast genomes but are vastly expanded in plants, thus supporting this hypothesis. Third, the genes coding for proteins of the PLD pathway may be preferentially multiplicated in some organisms relative to the gene repertoire of the PLC/DGK pathway so that the total supply

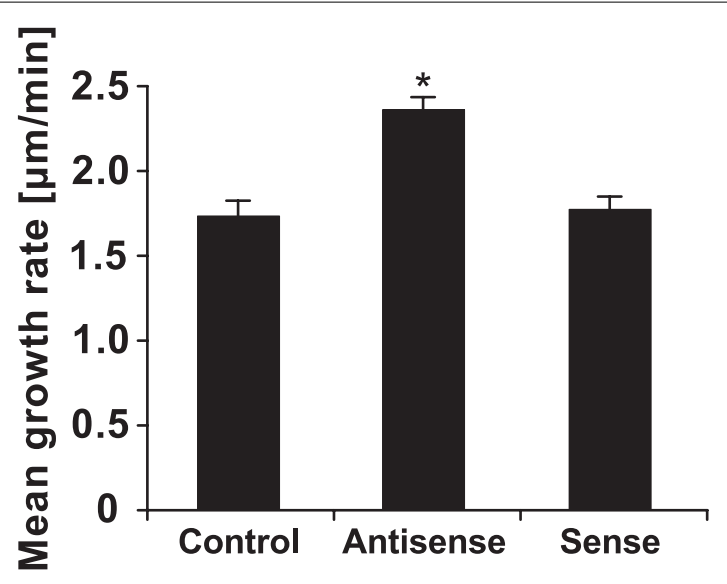

FIGURE 11 | Antisense ODNs-mediated knock-down of tobacco LPP4 promotes pollen tube growth. Pollen tubes were cultivated in the presence of $30 \mu \mathrm{M}$ antisense or sense ODNs against tobacco LPP4 for $90 \mathrm{~min}$. At least 100 cells were measured for each treatment in three independent experiments. Data show the mean growth rates of pollen tubes \pm SEM. The antisense-mediated promotion of the pollen tube growth rate is statistically significant in comparison with controls and sense ODNs (ANOVA, Kruskal-Wallis test, $p<0.05$ ).

of miscellaneous PA varieties for distinct signaling pathways is balanced. Such scenario is plausible in mammals whose genomes display much greater diversity of genes from the PLC/DGK pathway compared with "just” two PLD isoforms (Pleskot and Potocký, in preparation).

The involvement of PLD, DGK, and LPP in cellular processes can be investigated pharmacologically by application of several inhibitors that affect particular enzymes. This approach is especially beneficial when working with multi-gene families, of which PLDs, DGKs, and LPPs are typical examples. Based on our results, we speculate that one of the major targets of PLD-produced PA in the pollen tube is the actin cytoskeleton. The fact that PA plays an important role in the regulation of the actin cytoskeleton is further buttressed by the effect of PA itself and the PA phosphatase 
activity inhibitor NEM. Moreover, NEM behaved similarly to PA in all performed experiments including effects on vesicle trafficking, pectin deposition, and callose distribution. Other potential targets of PLD-derived PA may be considered. PA was implicated in the formation of a proper Golgi structure and function in both mammalian and plant cells (Langhans and Robinson, 2007; Riebeling et al., 2009), exocytosis (Zeniou-Meyer et al., 2007), and regulation of ROS production by activation of NADPH oxidase (Zhang et al., 2009). In addition, PA can directly modulate physical properties of membranes and influences membrane curvature because of its conical shape (Kooijman et al., 2003, 2005). The rapid induction of pollen tube growth and apical dynamics (Figure 9) may be partly assigned to this mechanism. PA can also stimulate SNARE-dependent membrane fusion in vitro (Vicogne et al., 2006).

Although Zonia and Munnik (2004) reported that the majority of PA in pollen tubes is produced via the PLD pathway and not the PLC/DGK pathway, the involvement of the PLC/DGK pathway for pollen tube elongation has not been previously tested. Recently, PLC activity was described to be a requisite for pollen tube growth (Pan et al., 2005; Dowd et al., 2006; Helling et al., 2006). Our results suggest that DGK is also involved in the regulation of tip growth. We observed a strong inhibition of pollen tube growth in response to treatment by the DGK inhibitor R59022. We labeled different cellular structures after treatment by $n$-ButOH and R59022 in order to find potential targets of distinct PA pools formed by PLD or DGK, respectively. We found the effect of R59022 on vacuolar morphology, which is specific for this DGK inhibitor. Vacuoles are crucial for the proper maintenance of turgor pressure and indirectly for pollen tube growth (Winship et al., 2010). The change in the vacuole dynamics could cause cessation of growth and other phenotypes (see FM 1-43 staining, Ruthenium red staining) visible after treatment by R59022. Another possible explanation is that DGK is important in the regulation of endomembrane trafficking. We observed non-impaired endocytosis after DGK inhibition, so it is plausible to hypothesize that further steps in the endosomal pathway are affected. A similar effect was described for the inhibition of phosphatidylinositol 3-kinase in the Arabidopsis root hairs (Lee et al., 2008). Also for animal cells, the involvement of several DGK isoforms in both clathrin-dependent endocytosis and endocytotic recycling has been described (Rincón et al., 2007; Kawasaki et al., 2008).

Inhibition of PA production in pollen tubes resulted in relocalization of FM 1-43 staining pattern into a funnel-like structure overlapping with the proposed zone for maximal endocytosis (Figure 4; also Derksen et al., 1995). However, early endocytosis of FM dyes into pollen tubes could not be completely stopped even with high concentrations of either $n$-ButOH or R59022. Similar results were described by Li and Xue (2007) who observed slower (but not stopped) endocytosis in Arabidopsis roots after PLD inhibition. Two explanations are possible. Either the residual amount of PA that is formed in $n$-ButOH/R59022-inhibited cells is sufficient for internalization or PA is not absolutely required for the first step of endocytosis. Nevertheless, the results described indicate that membrane cycling is disturbed in pollen tubes with inhibited PA production. Interestingly, the FM 1-43 staining pattern after $n$-ButOH treatment is strikingly different from patterns invoked by brefeldin A, cytochalasin D, latrunculin B, or sodium azide in pollen tubes and/or root hairs (Parton et al., 2001; Ovečka et al., 2005), indicating that some PLD or DGK isoform(s) may function in distinct recycling steps.

Experiments with inhibitors targeting LPP genes strongly suggest the involvement of the LPP isoform in the negative regulation of PA signaling in growing pollen tubes. This hypothesis was further tested by knock-down of the tobacco LPP4 by antisense ODNs, although it remains unclear if the pollen-selective LPP4 activity is specific to PA or to DGPP.

In summary, our results clearly demonstrate strikingly different roles of PA produced by PLDs and DGKs in pollen tube growth. We speculate that PA produced by PLD is more involved in the production of the exocytotic vesicles, their delivery to the pollen tube apex and their fusion with the plasma membrane. On the other hand, changes in the vacuole morphology and the appearance of pectin-containing punctuate compartments after the inhibition of DGK suggest the involvement of PA produced by DGK in the endocytotic processes. To address these hypotheses further, molecular studies on individual PA signaling genes will be necessary.

\section{MATERIALS AND METHODS PHYLOGENETIC ANALYSIS}

Eukaryotic PLD, DGK, and LPP protein sequences were identified by gapped BLAST or PSI-BLAST (Altschul et al., 1997) searching against the non-redundant protein database at the National Center for Biotechnology Information ${ }^{2}$ using Arabidopsis annotated sequences with default settings. In addition, blast searches were conducted via Phytozome website ${ }^{3}$. In most cases, the search parameters were set at the default values; however, occasionally, modifications were used (word size 2, scoring matrix BLOSUM45). We used AtPLD $\alpha 1$, AtDGK1, and AtLPP1 as input queries. Putative genes were initially identified based on the automatic annotation at the aforementioned databases. Since gene models based on computer annotations often contain errors, exon-intron structures were manually curated using SoftBerry server ${ }^{4}$ with the aid of experimentally verified sequences or sequences from closely related species.

Multiple alignments were constructed with mafft algorithms in einsi mode (Katoh and Toh, 2008) and manually adjusted. Conserved sequence blocks were concatenated giving alignments with 398 positions for PLDs, 348 positions for DGK, and 259 positions for LPPs. Maximum likelihood method using PhyML program (Guindon and Gascuel, 2003) was employed for phylogeny inference with the WAG matrix, $\gamma$-corrected among-site rate variation with four rate site categories plus a category for invariable sites, all parameters estimated from the data. Bayesian tree searches were performed using MrBayes 3.1 (Ronquist and Huelsenbeck, 2003) with a WAG amino-acid model, where all analyses were performed with four chains and 1,000,000 generations per analysis and trees sampled every 100 generations. All four runs asymptotically approached the same stationarity after first 500,000 generations which were omitted from the final analysis.

\footnotetext{
${ }^{2}$ http://blast.ncbi.nlm.nih.gov/Blast.cgi

${ }^{3}$ http://www.phytozome.net/

${ }^{4}$ http://linuxl.softberry.com/berry.phtml
} 
The remaining trees were used to infer the posterior probabilities for individual clades.

\section{POLLEN CULTURE}

Routinely, Nicotiana tabacum cv. Samsun pollen was cultured with a density of $2 \mathrm{mg} / \mathrm{ml}$ in simple sucrose medium [10\% (w/v) sucrose, $0.01 \%(\mathrm{w} / \mathrm{v})$ boric acid] as described previously (Potocký et al., 2003). Unless otherwise stated, pollen tube culture $90 \mathrm{~min}$ after the imbibition was supplemented with various substances. We used the final concentrations $0.5 \%(\mathrm{v} / \mathrm{v})$ of $n$-ButOH, $50 \mathrm{nM}$ NEM $(50 \mu \mathrm{M}$ stock solution in water), $50 \mu \mathrm{M}$ R59022 $(25 \mathrm{mM}$ stock solution in DMSO), and $50 \mu \mathrm{M}$ dioctanoyl-PA ( $5 \mathrm{mM}$ stock solution in water). Appropriate volumes of $t$-ButOH, medium, water and DMSO were used in control experiments. For confocal microscopy, pollen was cultivated on the rich medium solidified with $0.25 \%$ (w/v) phytagel according to Kost et al. (1998).

\section{FM 1-43 LABELING, VACUOLE, PECTIN AND CALLOSE STAINING, ACTIN LABELING}

Tobacco pollen tubes were labeled with endocytic marker FM 143 (Molecular Probes). After $90 \mathrm{~min}$ of cultivation, pollen tubes were put on ice and after $\sim 10$ min FM 1-43 dye at final concentration $2 \mu \mathrm{M}$ was simultaneously added with the inhibitors, PA, or control solutions into the germination medium. In an alternative arrangement of the experiment, pollen tubes were cultivated in a medium containing FM 1-43 for $90 \mathrm{~min}$, followed by treatment with particular inhibitor for $10 \mathrm{~min}$. Imaging was done on Zeiss LSM 5 DUO confocal laser scanning microscope using the Zeiss C-Apochromat 340/1.2 water corrected objective. A $489 \mathrm{~nm}$ laser line and a $505 \mathrm{~nm}$ long-pass emission filter were used for FM 1-43 imaging.

Vacuoles were labeled according to Lovy-Wheeler et al. (2007) with carboxy-(5-(and-6)-carboxy-2 $2^{\prime}, 7^{\prime}$-dichlorofluorescein diacetate) (carboxy-DCFDA) and imaging was performed on Zeiss LSM 5 DUO confocal laser scanning microscope using the Zeiss C-Apochromat 340/1.2 water corrected objective. A $489 \mathrm{~nm}$ laser line and a 505-550 $\mathrm{nm}$ band-pass emission filter were used for carboxy-DCFDA imaging.

Pectin and callose were visualized according to Ischebeck et al. (2008). Actin cytoskeleton was labeled by the method described previously (Lovy-Wheeler et al., 2005; Pleskot et al., 2010). Imaging was done on Olympus BX-51 microscope.

\section{VIDEO-ENHANCED CONTRAST MICROSCOPY AND TRANSMISSION ELECTRON MICROSCOPY}

Video microscopy was performed as described by Foissner et al. (1996). Briefly, the brightfield image from a Univar microscope (Reichert-Leica, Austria) equipped with a $40 \times$ planapo objective was collected with a high-resolution video camera (Chalnicon

\section{REFERENCES}

Altschul, S. F., Madden, T. L., Schäffer, A. A., Zhang, J., Zhang, Z., Miller, W., and Lipman, D. J. (1997). Gapped BLAST and PSI-BLAST: a new generation of protein database search programs. Nucleic Acids Res. 25, 3389-3402.
Arisz, S. A., and Munnik, T. (2011). The salt stress-induced LPA response in Chlamydomonas is produced via PLA2 hydrolysis of DGK-generated 2012-2020.

Arisz, S. A., Testerink, C., and Munnik, T. (2009). Plant PA signaling via phosphatidic acid. J. Lipid Res. 52,

C 1000.1, Hamamatsu, Germany), processed by a digital image processor (DVS 3000, Hamamatsu Germany), and recorded on digital video tape (JVC). Sequences were then converted to raw AVI movies using Adobe software and analyzed with ImageJ software.

For transmission electron microscopy, 50- to 80-min-old pollen tubes were fixed in $2 \%$ glutaraldehyde in $0.05 \mathrm{M}$ PIPES buffer at $\mathrm{pH} 7.2$ containing $5 \mathrm{mM} \mathrm{MgSO} 4$ and $5 \mathrm{mM}$ EGTA for $1 \mathrm{~h}$ at room temperature, followed by post-fixation in $1 \%(\mathrm{w} / \mathrm{v}) \mathrm{OsO} 4$ in water. Samples were dehydrated in ethanol and propylene oxide and embedded in Spurr's resin. For transmission electron microscopy, thin sections were post-stained with uranyl acetate and lead citrate according to standard procedures and viewed with a JEOL JEM 100CX II transmission electron microscope.

\section{CLONING OF TOBACCO LPP HOMOLOG, DESIGN OF ANTISENSE ODNs AND DELIVERY INTO GROWING POLLEN TUBES}

Total RNA from N. tabacum cv. Samsun pollen tubes was isolated using an RNeasy kit (Qiagen) and transcribed to cDNA using Transcriptor High Fidelity cDNA Synthesis Kit (Roche Applied Sciences) with $2.5 \mu \mathrm{g}$ RNA and oligo(dT) primers according to the manufacturer's instructions. Tobacco pollen LPP4 cds was amplified using Phusion DNA polymerase (Finnzymes) and degenerated primers forward, AGTGAATTCRATGCCGGAAATAGAATTTGG; reverse, ATTCTCGAGTTACACWGTGTCMATTSYTACTG. The PCR product mix was then cloned into pJET1.2 cloning vector (Fermentas) according to the manufacturer's instructions and sequenced. Cloned NtLPP4 sequence was deposited to GenBank database under the accession number JQ627633. DNA sequence of NtLPP4 was analyzed using Soligo software ${ }^{5}$ for suitable target regions for antisense ODNs. The two best-scoring antisense ODNs and corresponding sense control ODNs were synthesized with phosphorothioate modifications at both the $5^{\prime}$ and $3^{\prime}$ ends (four bases modified at each end). ODN/cytofectin complexes were prepared as described previously (Moutinho et al., 2001), and the pre-mix was added to the growth medium. Two antisense/sense ODNs pairs were tested for their effect on tobacco pollen tube growth, and the more effective pair was used for further experiments.

\section{ACKNOWLEDGMENTS}

This work was supported by the Grant Agency of the Academy of Sciences of the Czech Republic (GAAV project IAA601110916) and the Czech Ministry of Education, Youth and Sports (MSMT project 0021620858 and MSMT Research Centre LC06034). The authors express their thanks to the developers of Linux operation system and other open-source software used in preparation of this study, particularly Bibus, Gimp, Gnumeric, ImageJ, and Inkscape.

${ }^{5}$ http://sfold.wadsworth.org/soligo.pl

diacylglycerol kinase. Biochim. Biophys. Acta 1791, 869-875.

Bargmann, B. O. R., and Munnik, T. (2006). The role of phospholipase D in plant stress responses. Curr. Opin. Plant Biol. 9, 515-522.

Camacho, L., and Malhó, R. (2003). Endo/exocytosis in the pollen tube apex is differentially regulated by $\mathrm{Ca}^{2+}$ and GTPases. J. Exp. Bot. 54, 83-92.

Derksen, J. Rutten, T., Lichtscheidl, I. K., Dewin, A. H. N., Pierson, E. S., and Rongen, G. (1995). Quantitative-analysis of the distribution of organelles in tobacco pollen 
tubes - implications for exocytosis and endocytosis. Protoplasma 188, 267-276.

Donkor, J., Sariahmetoglu, M., Dewald, J., Brindley, D. N., and Reue, K. (2007). Three mammalian lipins act as phosphatidate phosphatases with distinct tissue expression patterns. J. Biol. Chem. 282, 3450-3457.

Dowd, P. E., Coursol, S., Skirpan, A. L., Kao, T., and Gilroy, S. (2006). Petunia phospholipase $\mathrm{C} 1$ is involved in pollen tube growth. Plant Cell 18, 1438-1453.

Eliáš, M., Drdová, E., Žiak, D., Bavlnka, B., Hála, M., Cvrcková, F., Soukupova, H., and Žárský, V. (2003). The exocyst complex in plants. Cell Biol. Int. 27, 199-201.

Eliáš, M., Potocký, M., Cvrčková, F., and Žárský, V. (2002). Molecular diversity of phospholipase D in angiosperms. BMC Genomics 3, 2. doi:10.1186/1471-2164-3-2

Ella, K. M., Meier, K. E., Kumar, A., Zhang, Y., and Meier, G. P. (1997). Utilization of alcohols by plant and mammalian phospholipase D. Biochem. Mol. Biol. Int. 41, 715-724.

Foissner, I., Lichtscheidl, I. K., and Wasteneys, G. O. (1996). Actinbased vesicle dynamics and exocytosis during wound wall formation in characean internodal cells. Cell Motil. Cytoskeleton 35, 35-48.

Furneisen, J. M., and Carman, G. M. (2000). Enzymological properties of the LPP1-encoded lipid phosphatase from Saccharomyces cerevisiae. Biochim. Biophys. Acta 1484 , 71-82.

Geitmann, A., and Steer, M. (2006). "The architecture and properties of the pollen tube cell wall," in The Pollen Tube, ed. R. Malhó (Berlin: Springer), 177-200.

Gómez-Merino, F. C., Arana-Ceballos, F. A., Trejo-Téllez, L. I., Skirycz, A., Brearley, C. A., Dörmann, P., and Mueller-Roeber, B. (2005). Arabidopsis AtDGK7, the smallest member of plant diacylglycerol kinases (DGKs), displays unique biochemical features and saturates at low substrate concentration: the DGK inhibitor R59022 differentially affects AtDGK2 and AtDGK7 activity in vitro and alters plant growth and development. J. Biol. Chem. 280, 34888-34899.

Gómez-Merino, F. C., Brearley, C. A., Ornatowska, M., Abdel-Haliem, M. E. F., Zanor, M., and Mueller-Roeber, B. (2004). AtDGK2, a novel diacylglycerol kinase from Arabidopsis thaliana, phosphorylates 1-stearoyl2-arachidonoyl-sn-glycerol and 1,2dioleoyl-sn-glycerol and exhibits cold-inducible gene expression. J. Biol. Chem. 279, 8230-8241.

Grobei, M. A., Qeli, E., Brunner, E., Rehrauer, H., Zhang, R., Roschitzki, B., Basler, K., Ahrens, C. H., and Grossniklaus, U. (2009). Deterministic protein inference for shotgun proteomics data provides new insights into Arabidopsis pollen development and function. Genome Res. 19, 1786-1800.

Grunt, M., Žárský, V., and Cvrčková, F. (2008). Roots of angiosperm formins: the evolutionary history of plant $\mathrm{FH} 2$ domain-containing proteins. BMC Evol. Biol. 8, 115. doi:10.1186/1471-2148-8-115

Guindon, S., and Gascuel, O. (2003). A simple, fast, and accurate algorithm to estimate large phylogenies by maximum likelihood. Syst. Biol. 52, 696-704.

Helling, D., Possart, A., Cottier, S., Klahre, U., and Kost, B. (2006). Pollen tube tip growth depends on plasma membrane polarization mediated by tobacco PLC3 activity and endocytic membrane recycling. Plant Cell 18, 3519-3534.

Ischebeck, T., Stenzel, I., and Heilmann, I. (2008). Type B phosphatidylinositol-4-phosphate 5-kinases mediate Arabidopsis and Nicotiana tabacum pollen tube growth by regulating apical pectin secretion. Plant Cell 20, 3312-3330.

Katoh, K., and Toh, H. (2008). Recent developments in the MAFFT multiple sequence alignment program. Brief. Bioinform. 9, 286-298.

Kawasaki, T., Kobayashi, T., Ueyama, T., Shirai, Y., and Saito, N. (2008). Regulation of clathrin-dependent endocytosis by diacylglycerol kinase delta: importance of kinase activity and binding to AP2alpha. Biochem. J. 409, 471-479.

Kooijman, E. E., Chupin, V., de Kruijff, B., and Burger, K. N. J. (2003). Modulation of membrane curvature by phosphatidic acid and lysophosphatidic acid. Traffic 4, 162-174.

Kooijman, E. E., Chupin, V., Fuller, N. L., Kozlov, M. M., de Kruijff, B., Burger, K. N. J., and Rand, P. R. (2005). Spontaneous curvature of phosphatidic acid and lysophosphatidic acid. Biochemistry 44, 2097-2102.

Kooijman, E. E., and Testerink, C. (2010). "Phosphatidic acid: an electrostatic/hydrogen-bond switch?" in Lipid Signaling in Plants, ed. T. Munnik (Berlin: Springer), 203-222.
Kost, B., Spielhofer, P., and Chua, N. H. (1998). A GFP-mouse talin fusion protein labels plant actin filaments in vivo and visualizes the actin cytoskeleton in growing pollen tubes. Plant J. 16, 393-401.

Kroeger, J. H., Daher, F. B., Grant, M., and Geitmann, A. (2009). Microfilament orientation constrains vesicle flow and spatial distribution in growing pollen tubes. Biophys. J. 97, 1822-1831.

Langhans, M., and Robinson, D. G. (2007). 1-Butanol targets the Golgi apparatus in tobacco BY-2 cells, but in a different way to Brefeldin A. $J$. Exp. Bot. 58, 3439-3447.

Lee, Y., Bak, G., Choi, Y., Chuang, W., Cho, H., and Lee, Y. (2008). Roles of phosphatidylinositol 3-kinase in root hair growth. Plant Physiol. 147, 624-635.

Li, G., and Xue, H. (2007). Arabidopsis PLDzeta2 regulates vesicle trafficking and is required for auxin response. Plant Cell 19, 281-295.

Lovy-Wheeler, A., Cárdenas, L., Kunkel, J. G., and Hepler, P. K. (2007). Differential organelle movement on the actin cytoskeleton in lily pollen tubes. Cell Motil. Cytoskeleton 64 217-232.

Lovy-Wheeler, A., Wilsen, K. L., Baskin, T. I., and Hepler, P. K. (2005). Enhanced fixation reveals the apical cortical fringe of actin filaments as a consistent feature of the pollen tube. Planta 221, 95-104.

Malhó, R., Liu, Q., Monteiro, D. Rato, C., Camacho, L., and Dinis, A. (2006). Signalling pathways in pollen germination and tube growth. Protoplasma 228, 21-30.

Martin, T. F. (1998). Phosphoinositide lipids as signaling molecules: common themes for signal transduction, cytoskeletal regulation, and membrane trafficking. Annu. Rev. Cell Dev. Biol. 14, 231-264.

McDermott, M., Wakelam, M. J. O., and Morris, A. J. (2004). Phospholipase D. Biochem. Cell Biol. 82, 225-253.

Meier, K. E., Gause, K. C., WisehartJohnson, A. E., Gore, A. C., Finley, E. L., Jones, L. G., Bradshaw, C. D., McNair, A. F., and Ella, K. M. (1998). Effects of propranolol on phosphatidate phosphohydrolase and mitogen-activated protein kinase activities in A7r5 vascular smooth muscle cells. Cell. Signal. 10, 415-426.

Mérida, I., Avila-Flores, A., and Merino, E. (2008). Diacylglycerol kinases: at the hub of cell signalling. Biochem. J. $409,1-18$

Monteiro, D., Castanho Coelho, P. Rodrigues, C., Camacho, L., Quader,
H., and Malhó, R. (2005). Modulation of endocytosis in pollen tube growth by phosphoinositides and phospholipids. Protoplasma 226, 31-38.

Moscatelli, A., Ciampolini, F., Rodighiero, S., Onelli, E., Cresti, M., Santo, N., and Idilli, A. (2007). Distinct endocytic pathways identified in tobacco pollen tubes using charged nanogold. J. Cell. Sci. 120, 3804-3819.

Moutinho, A., Camacho, L., Haley, A., Pais, M., Trewavas, A., and Malhó, R. (2001). Antisense perturbation of protein function in living pollen tubes. Sex. Plant Reprod. 14, 101-104.

Nakamura, Y., Tsuchiya, M., and Ohta, H. (2007). Plastidic phosphatidic acid phosphatases identified in a distinct subfamily of lipid phosphate phosphatases with prokaryotic origin. J. Biol. Chem. 282, 29013-29021.

Nakamura, Y., Koizumi, R., Shui, G., Shimojima, M., Wenk, M. R., Ito, T., and Ohta, H. (2009). Arabidopsis lipins mediate eukaryotic pathway of lipid metabolism and cope critically with phosphate starvation. Proc. Natl. Acad. Sci. U.S.A. 106, 20978-20983.

Nakamura, Y., and Ohta, H. (2010). "Phosphatidic acid phosphatases in seed plants," in Lipid Signaling in Plants, ed. T. Munnik (Berlin: Springer), 131-141.

Ohashi, Y., Oka, A., Rodrigues-Pousada, R., Possenti, M., Ruberti, I., Morelli, G., and Aoyama, T. (2003). Modulation of phospholipid signaling by GLABRA2 in root-hair pattern formation. Science 300, 1427-1430.

Ovečka, M., Baluška, F., and Lichtscheidl, I. (2008). Noninvasive microscopy of tip-growing root hairs as a tool for study of dynamic and cytoskeleton-based vesicle trafficking. Cell Biol. Int. 32, 549-553.

Ovečka, M., Lang, I., Baluška, F., Ismail, A., Illeš, P., and Lichtscheidl, I. K. (2005). Endocytosis and vesicle trafficking during tip growth of root hairs. Protoplasma 226, 39-54.

Pan, Y., Wang, X., Ma, L., and Sun, D. (2005). Characterization of phosphatidylinositol-specific phospholipase C (PI-PLC) from Lilium daviddi pollen. Plant Cell Physiol. 46, 1657-1665.

Parton, R. M., Fischer-Parton, S., Watahiki, M. K., and Trewavas, A. J. (2001). Dynamics of the apical vesicle accumulation and the rate of growth are related in individual pollen tubes. J. Cell Sci. 114, 2685-2695. 
Pleskot, R., Potocký, M., Pejchar, P., Linek, J., Bezvoda, R., Martinec, J., Valentová, O., Novotná, Z., and Žárský, V. (2010). Mutual regulation of plant phospholipase D and the actin cytoskeleton. Plant J. 62, 494-507.

Potocký, M., Eliáš, M., Profotová, B., Novotná, Z., Valentová, O., and Žárský, V. (2003). Phosphatidic acid produced by phospholipase $\mathrm{D}$ is required for tobacco pollen tube growth. Planta 217, 122-130.

Potocký, M., Jones, M. A., Bezvoda, R., Smirnoff, N., and Žárský, V. (2007). Reactive oxygen species produced by NADPH oxidase are involved in pollen tube growth. New Phytol. 174, 742-751.

Riebeling, C., Morris, A. J., and Shields, D. (2009). Phospholipase D in the Golgi apparatus. Biochim. Biophys. Acta 1791, 876-880.

Rincón, E., Santos, T., Avila-Flores, A., Albar, J. P., Lalioti, V., Lei, C., Hong, W., and Mérida, I. (2007). Proteomics identification of sorting nexin 27 as a diacylglycerol kinase zeta-associated protein: new diacylglycerol kinase roles in endocytic recycling. Mol. Cell. Proteomics 6, 1073-1087.

Ronquist, F., and Huelsenbeck, J. P. (2003). MrBayes 3: Bayesian phylogenetic inference under mixed models. Bioinformatics 19, 1572-1574.

Ruelland, E., Cantrel, C., Gawer, M., Kader, J., and Zachowski, A. (2002). Activation of phospholipases $\mathrm{C}$ and $\mathrm{D}$ is an early response to a cold exposure in Arabidopsis suspension cells. Plant Physiol. 130, 999-1007.

Šamaj, J., Ovečka, M., Hlavačka, A., Lecourieux, F., Meskiene, I.,
Lichtscheidl, I., Lenart, P., Salaj, J., Volkmann, D., Bögre, L., Baluška, H., and Hirt, H. (2002). Involvement of the mitogen-activated protein kinase SIMK in regulation of root hair tip growth. EMBO J. 21, 3296-3306.

Sun, C., Höglund, A., Olsson, H., Mangelsen, E., and Jansson, C. (2005). Antisense oligodeoxynucleotide inhibition as a potent strategy in plant biology: identification of SUSIBA2 as a transcriptional activator in plant sugar signalling. Plant $J$. $44,128-138$.

Testerink, C., and Munnik, T. (2011). Molecular, cellular, and physiological responses to phosphatidic acid formation in plants. J. Exp. Bot. 62, 2349-2361.

van Schooten, B., Testerink, C., and Munnik, T. (2006). Signalling diacylglycerol pyrophosphate, a new phosphatidic acid metabolite. Biochim. Biophys. Acta 1761, 151-159.

Vicogne, J., Vollenweider, D., Smith, J. R., Huang, P., Frohman, M. A., and Pessin, J. E. (2006). Asymmetric phospholipid distribution drives in vitro reconstituted SNARE-dependent membrane fusion. Proc. Natl. Acad. Sci. U.S.A. 103, 14761-14766.

Wang, X., Teng, Y., Wang, Q., Li, X., Sheng, X., Zheng, M., Samaj, J., Baluška, F., and Lin, J. (2006). Imaging of dynamic secretory vesicles in living pollen tubes of Picea meyeri using evanescent wave microscopy. Plant Physiol. 141, 1591-1603.

Wei, L. Q., Xu, W. Y., Deng, Z. Y., Su, Z., Xue, Y., and Wang, T. (2010). Genome-scale analysis and comparison of gene expression profiles in developing and germinated pollen in Oryza sativa. BMC Genomics 11, 338.

Winship, L. J., Obermeyer, G., Geitmann, A., and Hepler, P. K. (2010). Under pressure, cell walls set the pace. Trends Plant Sci. 15, 363-369.

Yamamoto, A., DeWald, D. B., Boronenkov, I. V., Anderson, R. A., Emr S. D., and Koshland, D. (1995). Novel PI(4)P 5-kinase homologue, Fablp, essential for normal vacuole function and morphology in yeast. Mol. Biol. Cell 6, 525-539.

Žárský, V., and Potocký, M. (2010). Recycling domains in plant cell morphogenesis: small GTPase effectors, plasma membrane signalling and the exocyst. Biochem. Soc. Trans. 38, 723-728.

Zeniou-Meyer, M., Zabari, N., Ashery, U., Chasserot-Golaz, S., Haeberlé, A., Demais, V., Bailly, Y., Gottfried, I., Nakanishi, H., Neiman, A. M., Du, G., Frohman, M. A., Bader, M., and Vitale, N. (2007). Phospholipase D1 production of phosphatidic acid at the plasma membrane promotes exocytosis of large dense-core granules at a late stage. J. Biol. Chem. 282, 21746-21757.

Zhang, Y., Zhu, H., Zhang, Q., Li, M., Yan, M., Wang, R., Wang, L., Welti, R., Zhang, W., and Wang, X. (2009). Phospholipase dalphal and phosphatidic acid regulate NADPH oxidase activity and production of reactive oxygen species in ABA-mediated stomatal closure in Arabidopsis. Plant Cell 21, 2357-2377.

Zonia, L. (2010). Spatial and temporal integration of signalling networks regulating pollen tube growth. $J$. Exp. Bot. 61, 1939-1957.
Zonia, L., and Munnik, T. (2004). Osmotically induced cell swelling versus cell shrinking elicits specific changes in phospholipid signals in tobacco pollen tubes. Plant Physiol. 134, 813-823.

Zonia, L., and Munnik, T. (2008). Vesicle trafficking dynamics and visualization of zones of exocytosis and endocytosis in tobacco pollen tubes. J. Exp. Bot. 59, 861-873.

Conflict of Interest Statement: The authors declare that the research was conducted in the absence of any commercial or financial relationships that could be construed as a potential conflict of interest.

Received: 01 December 2011; accepted: 29 February 2012; published online: 19 March 2012.

Citation: Pleskot R, Pejchar Pr, Bezvoda $R$, Lichtscheidl IK, Wolters-Arts M, Marc J, Žárský V and Potocký M (2012) Turnover of phosphatidic acid through distinct signaling pathways affects multiple aspects of pollen tube growth in tobacco. Front. Plant Sci. 3:54. doi: 10.3389/fpls.2012.00054

This article was submitted to Frontiers in Plant Physiology, a specialty of Frontiers in Plant Science.

Copyright (ㅇ 2012 Pleskot, Pejchar, Bezvoda, Lichtscheidl, Wolters-Arts, Marc, Žárský and Potocký. This is an open-access article distributed under the terms of the Creative Commons Attribution Non Commercial License, which permits non-commercial use, distribution, and reproduction in other forums, provided the original authors and source are credited. 